\title{
Radioactive seed and targeted axillary dissection: a feasibility study
}

\section{Rodriguez-Qizilbash S, Guilarte L, Lazizi S, Boulva K, Robidoux A, Younan R and Patocskai E Department of Surgery, Centre Hospitalier de l'Université de Montréal, Montreal, Canada}

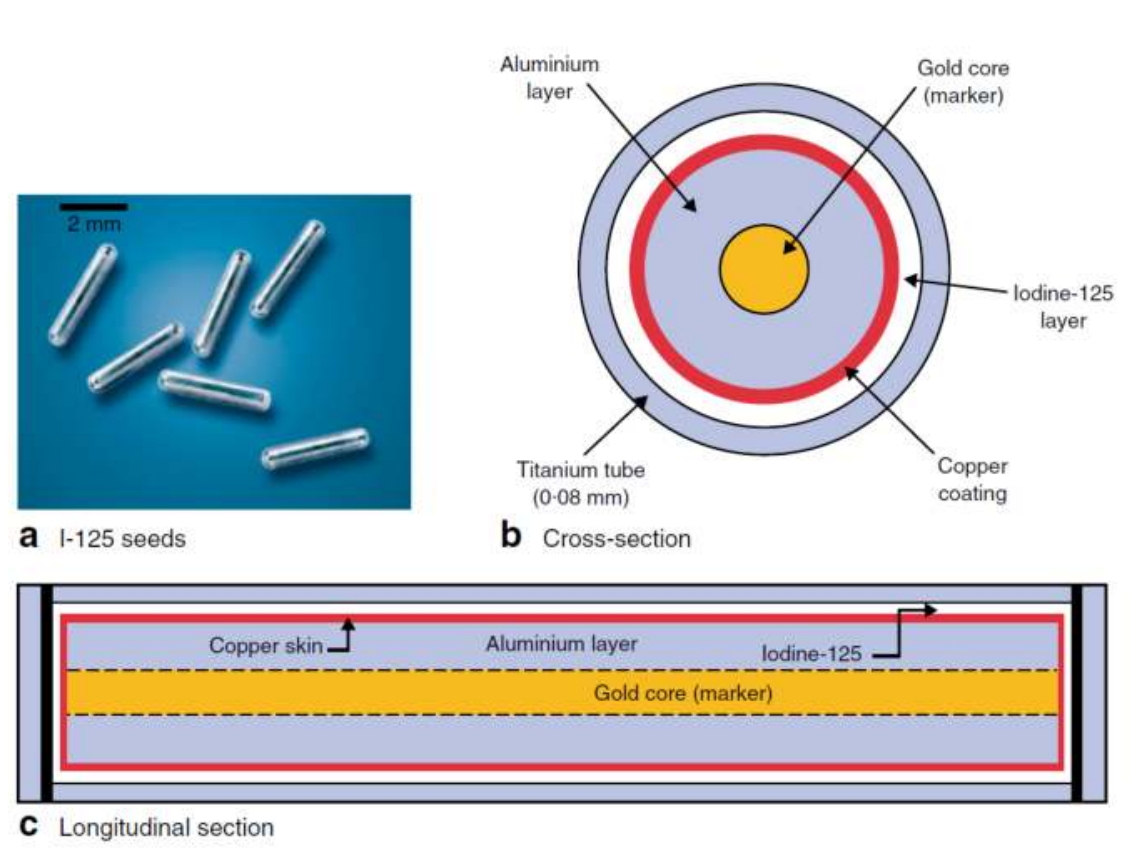

Composition of iodine-125-radiolabelled

(I-125) seeds Straver ME, et al., BJS 2010; 97: 1226-1231 (C) 2010 British Journal of Surgery Society Ltd

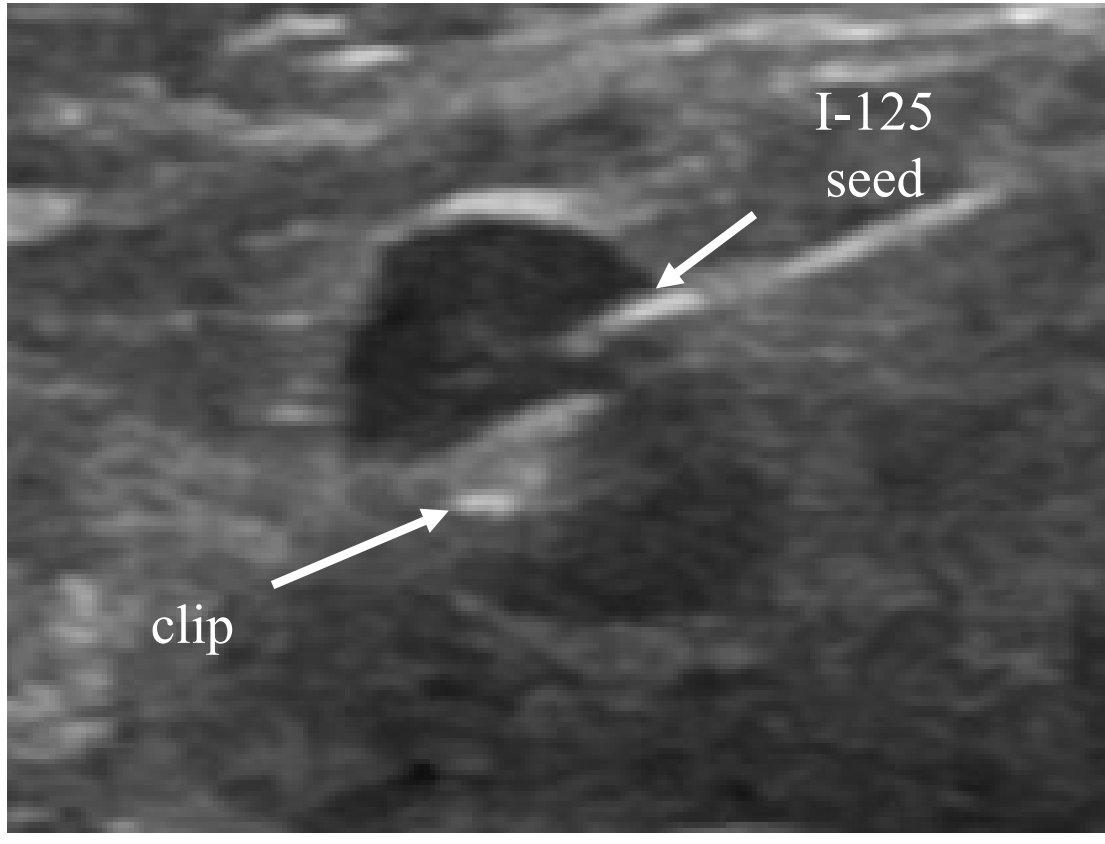

Ultrasound-guided insertion of the I-125 seed in a clipped positive node $\odot$ Erica Patocskai

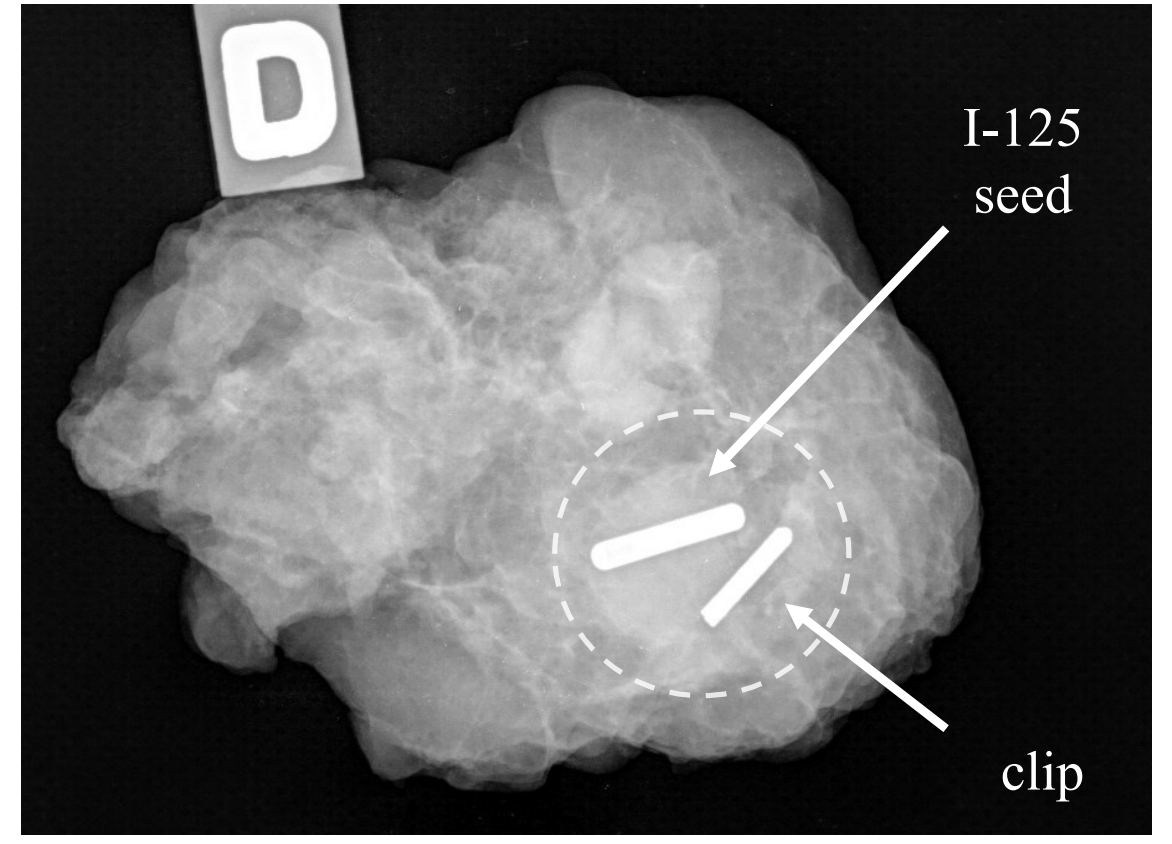

$X$-ray of an excised node containing a seed and a metallic clip $\odot$ Erica Patocskai

\section{INTRODUCTION}

Background: Targeted axillary dissection (TAD) with a radioactive seed is a new and promising technique to evaluate the axillary status in post-neoadjuvant chemotherapy (NACT) node-positive breast cancer patients.

Objective: This study aims to evaluate the feasibility of TAD with a radioactive seed in a Canadian setting.

\section{METHODS}

We conducted a retrospective observational study of a prospectively gathered database of patients having undergone TAD with a radioactive seed implanted in a proven metastatic axillary node, between 2015 and 2017. An iodine-125 radioactive seed was implanted under ultrasound guidance by trained radiologists.

Patients then underwent standard sentinel lymph node biopsy (SLNB) using technicium-99 and blue dye, as well as selective removal of the node containing the radioactive seed. Data was gathered from electronic medical records.

\section{DESCRIPTION OF THE TECHNIQUE}

1. Node-positive breast cancer patient candidate for neoadjuvant chemotherapy

2. Insertion of a metallic clip in the positive node.

3. Neoadjuvant chemotherapy

4. On the day prior to the operation, the radioactive seed is placed in the clipped node under ultrasound guidance.

5. The patient is brought to the operating room

6. Standard preparation for dual-tracer sentinel lymph node biopsy procedure

7. Standard sentinel lymph node biopsy + selective removal of the node with the radioactive seed (which can also be sentinel).

8. Specimen analyzed by pathology: the report states whether the node with the seed was metastatic and if it was also sentinel.

\section{RESULTS}

- Nine patients with a median age of 54 underwent TAD for unilateral breast cancer post-NACT

- Procedure performed between 2015 and 2017

- Triple negative breast cancer: $\mathbf{3 / 9}$

- median number of lymph nodes were removed, including that which contained the radioactive seed: $\mathbf{3}$

- Postoperative pathological reports:

- 4 patients with positive nodes

- 3 pathologic complete responses in the breast + axilla

- 2 patients with positive breast but complete response in the axilla

- The 4 patients with positive nodes post-neoadjuvant therapy had a seed in the positive node (no false negatives)

- The seed also located a positive retropectoral node that would otherwise have been missed using standard dual tracer SLNB.

- No complications due to the use of the radioactive seed were encountered.

- All implanted seeds were identified and retrieved within the pathology specimen.

\section{CONCLUSION}

- In patients with positive nodes post-neoadjuvant chemotherapy, the seed was implanted in a positive node (no false negatives)

- Combined to the sentinel lymph node biopsy, TAD seems to reflect the axillary status post-neoadjuvant chemotherapy.

- More research is required to evaluate the reproducibility of our results.

- Changes to seed composition are expected in the future: we hope that the passage from a radioactive to magnetic seed will increase adoption of this technique.

Contact information:

Erica Patocskai, Surgical Oncologist

Centre Hospitalier de l'Université de Montréal

Montreal, QC

Email: erica.patocskai.chum@ssss.gouv.qc.ca 